\title{
Formation and characterization of thiol-modified fibrillated whey protein isolate solution with enhanced functionalities
}

\begin{abstract}
The effect of thiolation using propanethiol on the functionalities of fibrillated whey protein isolate (WPI) solution at different $\mathrm{pH}$ values was studied. Fibrillated WPI solutions were thiolated at different molar ratios of propanethiol:carboxyl group $(0.5: 1,1: 1,2: 1,3: 1,4: 1)$ and the highest esterification extent ratio was obtained at 4:1 ( $\mathrm{pH} 9)$. We also found that the thiolation process improved the foaming capacity and foam stability. TEM micrographs evidenced aggregation of thiol-modified fibrillated WPI. A network of shortened fibrils attached to each other was formed upon thiolation, suggesting good physical interaction. This was coherent with the increment of zeta potential values, indicating a greater repulsion force to retard fibrils aggregation. Thiolation enhanced emulsifying stability index of thiolmodified fibrillated WPI solution $(\mathrm{pH} 8)$ and diminished its susceptibility to $\mathrm{pH}$ changes. This has broadened the potential application of fibrils as food ingredients.
\end{abstract}

Keyword: Esterification; Whey protein isolate; Emulsifying properties; Foaming properties 